\title{
GECCO'16 Model-Based Evolutionary Algorithms (MBEA) Workshop Chairs' Welcome
}

We would like to express our great pleasure in welcoming you to the GECCO Workshop on Model-Based Evolutionary Algorithms (MBEA Workshop'16), held at the GECCO 2016 International Conference.

Fixed, problem-independent variation operators often fail to effectively exploit important features of highquality selected solutions, potentially leading to inefficient optimization in cases where a performance advantage can be gained by using variation operators that are informed by learnable problem features. One way to make variation operators more powerful and flexible is to

- Model key features of solutions that influence their quality, and

- generate a new population of candidate solutions using the model in the expectation of improved solution quality.

When the model is a probability distribution, such evolutionary algorithms are commonly called estimation-of-distribution algorithms (EDAs). This includes such algorithms as PBIL, UMDA, CGA, ECGA, EBNA, LFDA, BOA, hBOA, PBIL_C, EGNA, EMNA, DEUM, AMaLGaM, CMA-ES, ACO and natural-gradient-based optimization algorithms, including NES and xNES.

EDAs in fact belong to a broader class of model-based evolutionary algorithms (MBEA) that learn and store more general structure such as linkage, variable dependency structures and hypergraphs or that operate on ensembles of models. Examples include LTGA and DSMGA(-II) which do not construct a probabilistic model. Such algorithms have the potential to be more robust to changes in problem formulation, making them generally more attractive to solve black-box optimization problems.

Conversely, since their search trajectories are determined by explicit models, MBEAs are more amenable to theoretical study including approaches such as run-time analysis. Understanding gained here can lead to more principled algorithm design, informed selection of suitable representations and generalisation beyond empirical benchmark testing.

With the ending of the EDA track in the general GECCO conference, the focus on MBEA potentially becomes scattered across different tracks. The purpose of this workshop is therefore to provide a unique forum to discuss

- recent advances in model-based evolutionary algorithms

- new theoretical and empirical results,

- applications of model-based evolutionary algorithms,

- cross-fertilization between domains and techniques, and

- promising directions for future research.

We would like to end by thanking the invited speakers for their contributions.

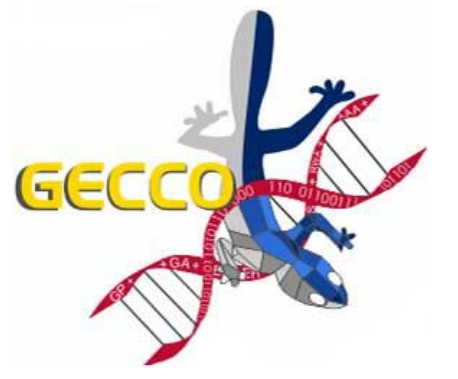

Peter A.N. Bosman

John McCall

Centrum Wiskunde \&

Robert Gordon University

Informatica, The Netherlands 